\title{
Prevalence, treatment patterns, and risk factors of hypertension and pre-hypertension among Bangladeshi adults
}

\author{
Mujibur Rahman ${ }^{1} \cdot$ M. Mostafa Zaman ${ }^{2}$. Jessica Yasmine Islam ${ }^{3}$. Jalil Chowdhury ${ }^{4}$ HAM Nazmul Ahsan ${ }^{1}$. \\ Ridwanur Rahman $^{5} \cdot$ Mahtabuddin Hassan $^{6} \cdot$ Zakir Hossain $^{7} \cdot$ Billal Alam $^{1} \cdot$ Rubina Yasmin $^{1}$
}

Received: 5 June 2017 / Revised: 3 October 2017 / Accepted: 16 October 2017 / Published online: 11 December 2017

(c) The Author(s) 2017. This article is published with open access

\begin{abstract}
In Bangladesh, morbidity and mortality due to non-communicable diseases (NCDs) has increased over the last few decades. Hypertension is an important risk factor for NCDs, specifically cardiovascular disease. The objective of this study was to assess prevalence and risk factors for hypertension and pre-hypertension among adults in Bangladesh. Data for this analysis were collected during the national NCD Risk Factor Survey of Bangladesh conducted in 2010 from a representative sample of men and women, aged 25 years or above. The survey adopted a multistage, geographically clustered, probability-based sampling approach. WHO STEPS questionnaire was used to collect data on demographics, behavioral risk factors, and physical measurements. Overall, $20 \%$ of the study population were hypertensive at study measurement. The prevalence of hypertension increased with age and body mass index (BMI). Twelve percent of the population were previously diagnosed with hypertension. Among these individuals, nearly half were not taking any medications to control their hypertension. Additionally, the prevalence of pre-hypertension was $43 \%$, with higher levels among males, older age groups, and those with higher education, higher wealth index and high BMI. Predictors of hypertension, included older age, high BMI, and diabetes comorbidity. Based on this study, we estimate that 1 out of 5 Bangladeshi adults have hypertension. The risk of hypertension increases with older age and high BMI. Additionally, prevalence of pre-hypertension is high in Bangladesh in both rural and urban areas. Findings from this study can be used to inform public health programming to control the spread of NCDs in Bangladesh.
\end{abstract}

\section{Introduction}

Globally, cardiovascular disease (CVD) results in 17 million deaths a year, nearly one-third of all deaths [1].

M. Mostafa Zaman

zamanm@who.int

1 Dhaka Medical College \& Bangladesh Society of Medicine, Dhaka, Bangladesh

2 Research and Publication, World Health Organizations, Dhaka, Bangladesh

3 Department of Epidemiology, University of North Carolina at Chapel Hill, Chapel Hill, NC, USA

4 BSMMU \& Bangladesh Society of Medicine, Dhaka, Bangladesh

5 Shaheed Suhrawardy Medical College \& Bangladesh Society of Medicine, Dhaka, Bangladesh

6 Chittagong Medical College \& Bangladesh Society of Medicine, Dhaka, Bangladesh

7 Rangpur Medical College \& Bangladesh Society of Medicine, Dhaka, Bangladesh
Hypertension is the most widely prevalent, largely preventable risk factor for death and disability worldwide due to CVDs [2, 3]. Globally, 7.7 million annual premature deaths and $6 \%$ of the global burden of disease is attributable to hypertension [4]. Pre-hypertension or elevated blood pressure (BP) lower than the standard cutoff for hypertension [systolic blood pressure (SBP) 140/diastolic blood pressure (DBP) $90 \mathrm{~mm} \mathrm{Hg}$ ] is also associated with increased risk of myocardial infarction, stroke, and CVD [5]. As such, the growing prevalence of hypertension is a major public health concern. The global prevalence of hypertension is projected to increase from $26 \%$ in 2000 to $29.2 \%$ by 2025 , which will be approximately $29 \%$ of the world's population [3]. This increase in hypertension is specifically notable among South Asian countries [6] and other low- and middle-income countries. The epidemic of hypertension and related comorbidities such as diabetes and stroke, will account for the greatest burden of death, disability, and health expenditures in the near future in these regions [7].

The burden of hypertension has shifted to largely effect low-income and developing countries, instead of developed 
countries such as the United States. In recent years, rapid urbanization, increased life expectancy, unhealthy diet, and lifestyle changes have led to an increase in the rate of CVD and its risk factors, including hypertension, in low- and middle-income countries, including Bangladesh [8]. This epidemiological transition in disease profile has a significant impact in low resource settings with weak health systems, as the number of people with hypertension who are diagnosed, untreated, and uncontrolled are also higher in low- and middle-income countries compared to high-income countries [1]. This shift in burden can be illustrated in Bangladesh where the first documented prevalence of hypertension was $1.10 \%$ in 1976 [9]. Recent studies have found the prevalence of hypertension to be as high as $26.4 \%$ among adults, with a disproportionate burden among females compared to males [10]. The prevalence of hypertension in Bangladesh has varied in the literature depending on the sample size and population characteristics, however, all findings consistently support the observation that prevalence of hypertension is on the rise among Bangladeshi adults [11-14]. Of particular value is to assess those at most risk of developing hypertension, the pre-hypertensive population, as hypertension can be prevented with timely administration of anti-hypertensive medications [15]. Data of a nationally representative sample are needed to provide accurate statistics on prevalence of hypertension and pre-hypertension, and associated risk factors by various sociodemographic factors such as sex and area of residence to inform future public health initiatives tailored to specific target population.

The present study was conducted as a part of the nationwide survey to assess risk factors for non-communicable diseases in Bangladesh. In this report, we assessed the prevalence, treatment patterns, and control of hypertension. Additionally, we explored risk factors of hypertension among our study sample of Bangladeshi adults. As a secondary aim, we explored the prevalence of pre-hypertension in this population and associated risk factors.

\section{Methods}

This assessment was conducted as a part of the national survey on non-communicable disease (NCD) risk factors conducted by the Bangladesh Society of Medicine, under the guidance of the Directorate General of Health Services, and with technical assistance from the WHO Country Office for Bangladesh. It was a population-based, cross-sectional study carried out from November 2009 to April 2010 following the WHO STEPwise Surveillance (STEPS) approach. The target population of this survey was male and females aged 25 years and above residing in rural and urban areas of Bangladesh. Individuals who were institutionalized, including people residing in hospitals, prisons, nursing homes, etc. were excluded from this study. Written consent (or thumb impression) was obtained before participants were enrolled in the study. International ethical guidelines for biomedical research involving human subjects were followed throughout the study and approved by an independent Ethical Research Committee (ERC) of Bangladesh Medical Research Council (BMRC).

\section{Sampling methods}

The study adopted a multistage, geographically clustered, probability-based sampling approach to produce nationally representative data for Bangladesh. The sampling frame was updated by the Bangladesh Bureau of Statistics in 2009 . Data were collected from 200 mahalla (urban areas) and 200 mauza (rural areas) from 62 districts of Bangladesh, using digital technology.

The ultimate sampling units were the household and one individual (equal number of men and women) residing in the selected household. Sampling of eligible individuals was done from a sample of households with one individual randomly selected per household. Each selected household was randomly assigned as a men or women household. The power analysis and sample size calculations were completed based on the standardized approach devised by the World Health Organization (WHO) known as STEP-wise approach to Surveillance of NCD risk factors (STEPS) [16]. Using the prevalence of tobacco use of $43.3 \%$ within the population [17] and 3\% margin of error, and assuming a design effect of 2, the minimum sample size was 2096 (rounded to 2100). This was multiplied by 4 to get a national estimate for four groups (men, women, urban areas of residence, rural areas of residence). Therefore, a minimum of 8400 respondents was needed. Considering a response of $84 \%$, the sample size was inflated to $10,000(8400 \times 0.84)$. Then, to get 10,000 respondents, 25 households per PSU were targeted. A total of 9947 individuals from 10,991 households were approached to participate in the survey. Overall, 9275 (4312 men and 4963 women) non-institutionalized adults aged $\geq 25$ years agreed to participate, leading to a response rate of 93.2\%. Further details of the survey implementation and methodology have been previously reported [18].

\section{Survey instrument}

The national NCD risk factor survey was conducted using a standardized questionnaire and physical examination. The questionnaire was developed using the WHO STEPwise questionnaire [19], with minor adaptations and translated to Bengali from English. Data on sociodemographic and behavioral risk factors were collected during STEP 1. The household component of the questionnaire included a 20 -item index to assess assets and wealth. Information on 
hypertension was collected from all participants. Relevant information on age, area of residence, education, current occupation, eating habits and tobacco use was also collected. Physical measurements such as height, weight, waist circumference, and blood pressure were collected during STEP 2. Data on current high blood pressure were collected through blood pressure measurements taken on study site. Participants were asked if they had been previously diagnosed with high blood pressure based on self-report. Relevant information was obtained from medical records or other authentic documents as necessary and when available from study population.

Blood pressure was measured by appropriately calibrated aneroid sphygmomanometer by a trained field interviewer using appropriately sized arm cuffs. Blood pressure measurements were consistently taken on the right arm at level of the heart and elbow-assisted. The initial measurement was performed after $5 \mathrm{~min}$ of rest on the right arm. After $2 \mathrm{~min}$, the second measurement was taken. The Korotkoff phase I (beginning of the sound) and the phase V of Korotkoff (disappearance of the sound) was recorded as systolic and diastolic blood respectively. The mean of these two blood pressure readings was utilized as the final blood pressure for each participation.

As per the WHO STEPwise core questions on hypertension, participants were asked about their history of high blood pressure through the following questions: (1) "Have you ever had your blood pressure measured by a doctor or other health worker?"; (2) "Have you ever been told by a doctor or other health-care professional that you have high blood pressure?"; and (3) "Have you been told in the past 12 months?" Previous treatment and treatment patterns for hypertension were assessed utilizing the following questions: (1) "Are you currently receiving any of the following treatment/advice for high blood pressure prescribed by a doctor or other health workers? (a) Drugs/ medication that you have taken in the past 2 weeks, (b) Advised to reduce salt intake, (c) Advised or treated to lose weight, (d) Advised or treated to stop smoking, and (e) Advised to start or do more exercise."

\section{Outcome}

Our primary outcomes of interest were overall prevalence of hypertension and of pre-hypertension. We utilized the American Heart Association (AHA) guidelines for cutoff points to define hypertension. An individual was considered to have hypertension if SBP was $\geq 140 \mathrm{~mm} \mathrm{Hg}$ (millimeters of mercury) and/or, DBP $\geq 90 \mathrm{~mm} \mathrm{Hg}$ and/or taking any-hypertensive medication during survey based on selfreport. Pre-hypertension was defined as SBP $\geq 120 \mathrm{~mm} \mathrm{Hg}$ but $<140 \mathrm{~mm} \mathrm{Hg}$ and/or DBP $\geq 80 \mathrm{~mm} \mathrm{Hg}$ but $<90 \mathrm{~mm} \mathrm{Hg}$ and not taking anti-hypertensive medication at the time of the survey.

\section{Covariates}

The following variables were used as risk factors: sex, age, education, occupation, wealth index, body mass index (BMI), alcohol use, diabetes, and tobacco use. Education was categorized into five groups: no education, less than primary (completed grade 4 and below), primary school (completed grade 5), secondary school (completed grade 10 ), and higher secondary and above (completed grade 12 and above). Due to fewer frequencies, tertiary or university level education was not categorized into a separate group. Occupation was categorized into five groups for analysis. These groups included: professional employment (government employee, non-government employee, business owner, farmer, agricultural worker, and other selfemployed), unemployed or retired, industrial worker or day laborer, housemaker and other (beggar, rickshaw puller, cook, carpenter, tailor, security guard, migrant workers, and fishermen). The wealth index was constructed using principal component analysis. Asset information collected covered information on household ownership several items, including: electricity, flush toilet, land telephone, cell phone, television, radio, refrigerator, car, moped/scooter/ motor cycle, washing machine, bicycle, sewing machine, almirah/wardrobe, table, bed or cot, chair or bench, watch or clock, as well as the type of main material used for the roof, wall and floor of the main house (cement, tin, or bamboo/thatched/straw). Each asset was assigned a weight (factor score) generated through principal components analysis, and the resulting asset scores were standardized in relation to a normal distribution with a mean of zero and standard deviation of one. Each household was then assigned a score for each asset, and the scores were summed for each household; individuals were ranked according to the total score of the household in which they resided. The sample was then divided into quartiles from one (lowest) to four (highest). A single asset index was developed for the whole sample; indices were not prepared for urban and rural populations separately.

Utilizing height (in centimeters) and weight (in kilograms) measurements taken during study data collection, we calculated BMI (height/weight ${ }^{2}$ ) and categorized these measurements to the following groups: underweight ( $\leq 18.50)$, normal (18.6-25), overweight (25.1-30), and obese $(>30)$. Inadequate fruit and/or vegetable intake was defined as less than five servings per day based on selfreport. One standard serving size was defined to the participants as 80 grams and described using pictorial show cards or measuring cups. Alcohol use was defined as ever consumption of alcohol based on self-report. Diabetes was defined based on self-report of taking diabetes medication at the time of the study. We categorized participants as tobacco users if they either smoked cigarettes, cigars, or 
pipes, or if they used smokeless tobacco products such as zarda, sadapata, gul, and/or snuff based on self-report.

\section{Data analysis}

Sociodemographic variables were presented with median (interquartile range) for continuous variables and with proportion for categorical variables. The overall, sex-specific, age-specific by sex, and area-specific prevalence of hypertension were calculated. For bivariate analyses, study participants were divided by sex and into five age groups (25-34, 35-44, 45-54, 55-64, and 65+ years). A chi-square test was performed to assess proportional differences in hypertension and treatment patterns across select categorical variables. Adjusted and unadjusted logistic regression analyses were performed to identify significant predictors, or risk factors, of pre-hypertension, and hypertension. Potential risk factors were assessed using bivariate logistic regression analysis; an arbitrary $p$-value of $<0.20$ was used as criteria to include the variable in the multivariable logistic regression model to control for confounding effects. For multivariable logistic regression models, crude odds ratios (cOR), adjusted odds ratios (aOR), and 95\% confidence intervals (CI) for each independent variable were calculated, $p<0.05$ was used as the level of significance. Multivariable logistic regression models were generated separately for urban and rural participants to account for possible effect measure modification. The outcome variable of the model to identify risk factors for hypertension was coded as " 1 " if the participant was found to be hypertensive at study measurement based on the definition described above and the rest were coded as " 0 ." To identify predictors of pre-hypertension, we excluded those with existing hypertension at study measurement from the binary dependent variable of pre-hypertension. Accordingly, the outcome variable was coded as " 1 " if the participant was found to be pre-hypertensive at study measurement and the rest were coded as "0." All statistical procedures were performed using Stata/SE 12.1 (StataCorp LP, Texas, USA) software package.

\section{Results}

\section{Background characteristics}

Table 1 provides a summary of sociodemographic statistics of the study population. Overall, 9275 adults were included in this study sample from both urban $(n=4629)$ and rural $(n=4646)$ region of the country (Table 1$)$. Of these, $46.5 \%$ $(n=4312)$ were male. The median age and education level

Table 1 Sociodemographic factors and comorbidities among 9275 Adults in the Non-Communicable Disease Risk-Factor Survey of Bangladesh, 2010

\begin{tabular}{|c|c|c|c|c|c|c|c|c|c|}
\hline \multirow[b]{2}{*}{ Characteristic } & \multicolumn{3}{|c|}{ Male $(n=4312)$} & \multicolumn{3}{|c|}{ Female $(n=4963)$} & \multicolumn{3}{|c|}{ Total $(n=9275)$} \\
\hline & $\begin{array}{l}\text { Median } \\
\text { (IQR) }\end{array}$ & No. & $\%$ & $\begin{array}{l}\text { Median } \\
\text { (IQR) }\end{array}$ & No. & $\%$ & $\begin{array}{l}\text { Median } \\
\text { (IQR) }\end{array}$ & No. & $\%$ \\
\hline Age (years) & $43(33,55)$ & - & - & $38(30,47)$ & - & - & $40(31,50)$ & - & - \\
\hline Education (years) & $5(0,9)$ & - & - & $3(0,7)$ & - & - & $3(0,8)$ & - & - \\
\hline Urban residence & & 2175 & 50.4 & & 2454 & 49.5 & & 4629 & 49.9 \\
\hline $\begin{array}{l}\text { Body mass index } \\
(\mathrm{BMI})^{\mathrm{a}}\end{array}$ & $\begin{array}{l}20.1(18.3 \\
22.9)\end{array}$ & - & - & $\begin{array}{l}21.3(18.7 \\
24.5)\end{array}$ & - & - & $\begin{array}{l}20.7(18.4 \\
23.8)\end{array}$ & - & - \\
\hline $\begin{array}{l}\text { Waist circumference } \\
(\mathrm{cm})\end{array}$ & $76(70,83)$ & - & - & $80(73,86)$ & - & - & $78(72,84)$ & - & - \\
\hline Overweight or Obese ${ }^{b}$ & & 561 & 13 & & 1074 & 21.6 & & 1635 & 17.6 \\
\hline $\begin{array}{l}\text { Fruit consumption } \\
\text { (servings /day) }\end{array}$ & $1(0,1.0)$ & - & - & $1(0,2.0)$ & - & - & $1(0,1.5)$ & - & - \\
\hline $\begin{array}{l}\text { Vegetable } \\
\text { consumption (servings/ } \\
\text { day) }\end{array}$ & $2(1,2.5)$ & - & - & $\begin{array}{l}2.0(1.0, \\
3.0)\end{array}$ & - & - & $2(1,3)$ & - & - \\
\hline Tobacco use ${ }^{c}$ & - & 3020 & 70.4 & - & 1709 & 34.3 & - & 4729 & 51 \\
\hline Ever alcohol use $^{\mathrm{d}}$ & - & 504 & 11.7 & - & 15 & 0.3 & - & 519 & 5.6 \\
\hline Prevalence of diabetes ${ }^{\mathrm{e}}$ & - & 559 & 13 & - & 690 & 13.9 & - & 1249 & 13.5 \\
\hline
\end{tabular}

\footnotetext{
${ }^{a}$ Body Mass Index (BMI) calculated by weight in kilogram divided by height in meter squared; 56 missing values

${ }^{\mathrm{b}}$ Defined as $\mathrm{BMI} \geq 25$

${ }^{\mathrm{C}}$ Includes smoking and smokeless tobacco

${ }^{\mathrm{d}}$ Defined as alcohol consumption within the past 30 days

${ }^{\mathrm{e}}$ Based on self-report
} 
of participants was $40(31,50)$ years and $3(0,8)$ years, respectively. Overall, the median BMI was 20.7 (18.4, 23.8). The proportion of overweight or obesity was higher among females $(21.6 \%)$ than males (13\%). Fruit and vegetable consumption was very low among the total population with only a median $1(0,1.5)$ and $2[1,3]$ servings per day of fruit and vegetables, respectively. Half of participants reported to use some form of tobacco (smoke and/or smokeless tobacco) and 5.6\% self-reported to ever use alcohol. The prevalence of diabetes was $13.5 \%$ among our study population.

The average systolic and DBP was measured at 119.8 $\mathrm{mm} \mathrm{Hg}$ and $76.4 \mathrm{~mm} \mathrm{Hg}$, respectively. Among both males and females, an increase in trend by age groups was observed for systolic blood pressure. Similarly, an increase in trend of diastolic blood pressure was observed in both sexes till age 55-64 years. A decrease was then observed, specifically among females, among those aged 65 years and above (Fig. 1).
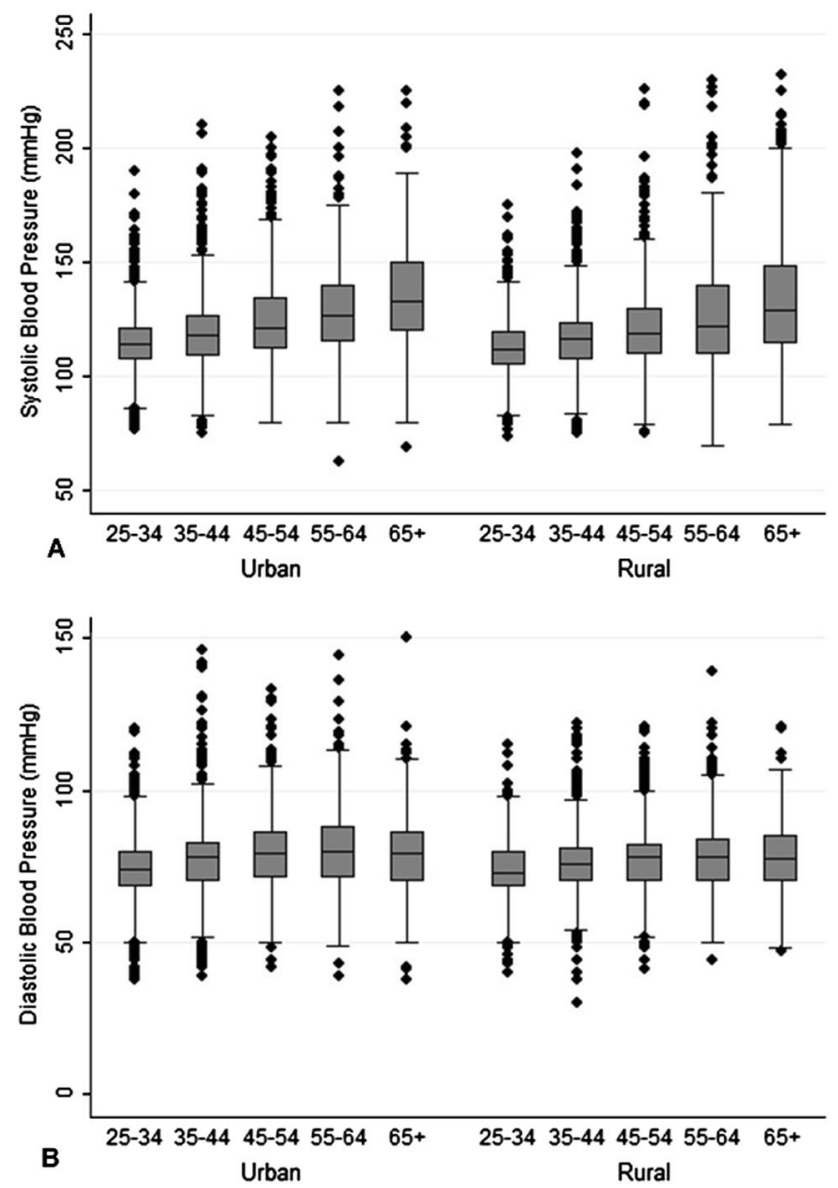

Fig. 1 Systolic blood pressure (a) and diastolic blood pressure (b) by age group among 9275 Bangladeshi adults, 2010
Prevalence, treatment patterns, and risk factors for hypertension

The prevalence of hypertension at study measurement was $20.1 \%$ with no significant difference between males $(20.3 \%)$ or females $(19.9 \%)$ (Table 2). However, the prevalence of hypertension increased by age group $(p<0.001)$ with the highest prevalence of hypertension to be among those aged 65 years and above among both males (40.5\%) and females $(50.5 \%)$. The prevalence of hypertension was higher among those residing in urban areas $(22.2 \%)$ than those in rural areas (17.9\%) (Table 3). Additionally, the prevalence of hypertension was highest across all age groups among those in the 4th (highest) wealth quartile (Fig. 2).

Among this study population, 6225 (67.1\%) reported to previously have their blood pressure taken by a health-care worker (Table 2). This proportion was higher among females $(77.2 \%)$ than males (55.6\%). Of these participants, $18.6 \%(n=1156)$ were previously diagnosed with high blood pressure or hypertension. Additionally, less than half $(42.5 \%, n=490)$ of those previously diagnosed were diagnosed in the past twelve months. Among participants who were previously diagnosed with hypertension or high blood pressure, $52.3 \%$ were currently taking prescription medications to control their hypertension. This proportion was lower among females (48.6\%) than males (57.9\%), however, increased with age $(p<0.001)$ with $68 \%$ of participants aged 65 years and above taking hypertensive medications. Among this older study population, $65.3 \%$ of men and $77.10 \%$ of women were diagnosed with hypertension at study measurement despite their previous diagnosis (Fig. 3).

Table 3 presents results of the multivariable logistic regression analysis to identify predictors associated with diagnosis of hypertension at study measurement by area of residence, together with adjusted and crude odds ratios, and 95\% confidence intervals after adjusting for a number of important covariates. Among urban participants, those with older age, wealth index in the 4th wealth quartile, increasing BMI and with diabetes were more likely to have hypertension. The odds of hypertension were significantly highest among those aged 65 years and above (aOR 14.4, 95\% CI: 10.0-20.5) compared to individuals aged 25-34 years of age. The odds of hypertension among those in the 4th wealth quartile was $40 \%$ higher than individuals in the 1 st wealth quartile (aOR: 1.4, 95\% CI: 1.0-1.9). The odds of having hypertension increased as BMI increased. For urban individuals with diabetes, the odds of having hypertension were $61 \%$ (aOR 1.6, 95\% CI: 1.3-1.9) higher than individuals without diabetes.

Similar trends were identified for rural participants, specifically for increasing age and BMI. The odds of having 


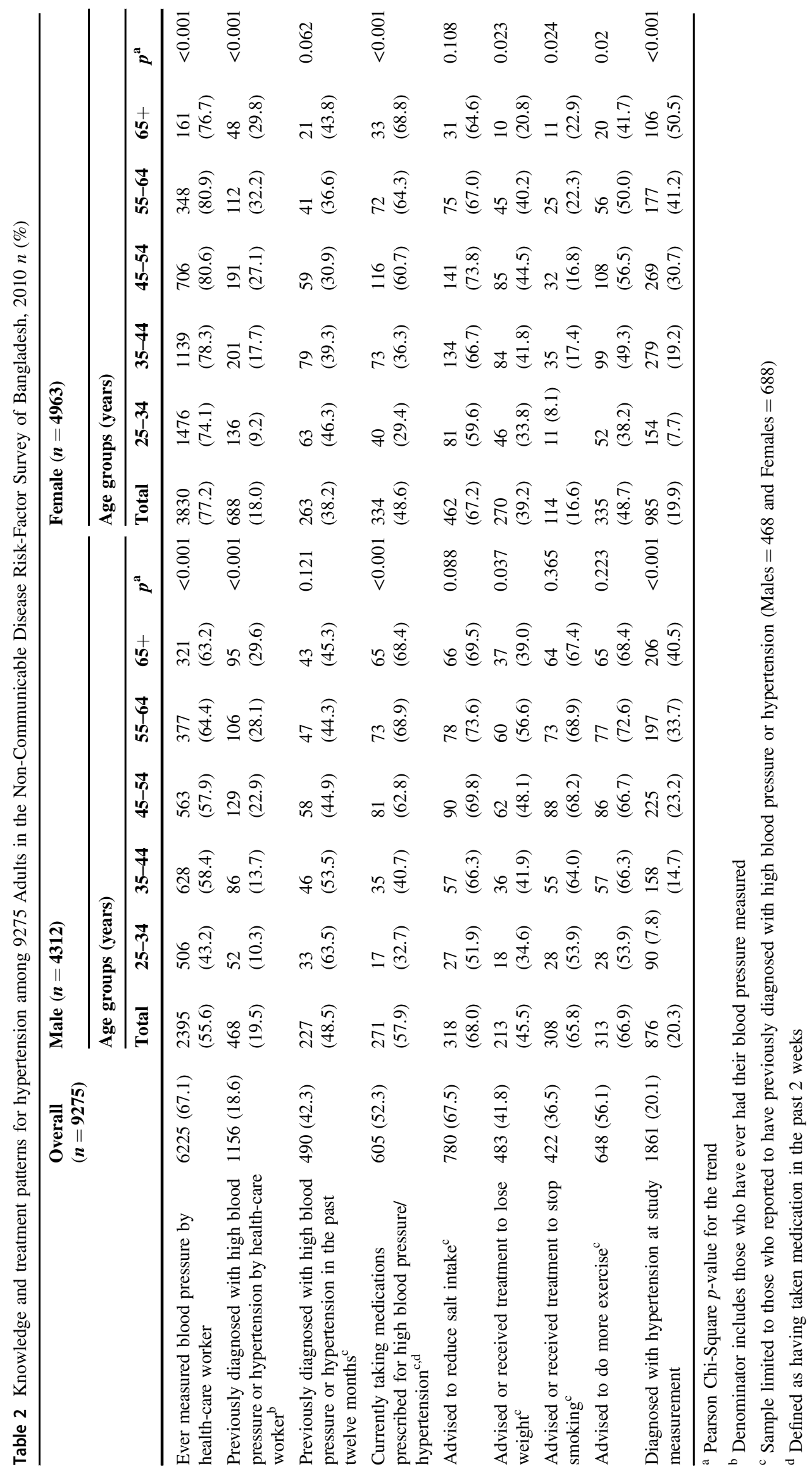




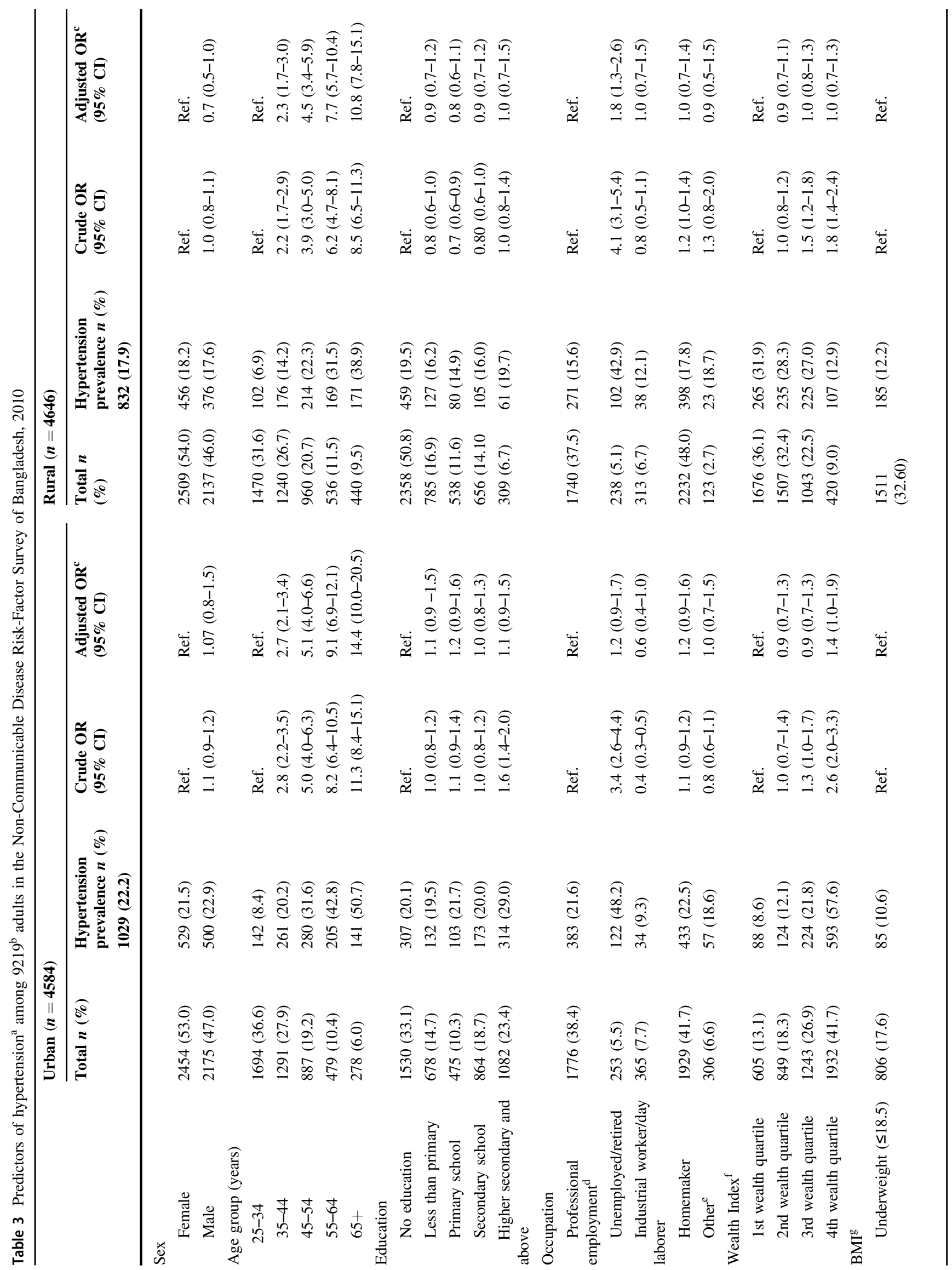




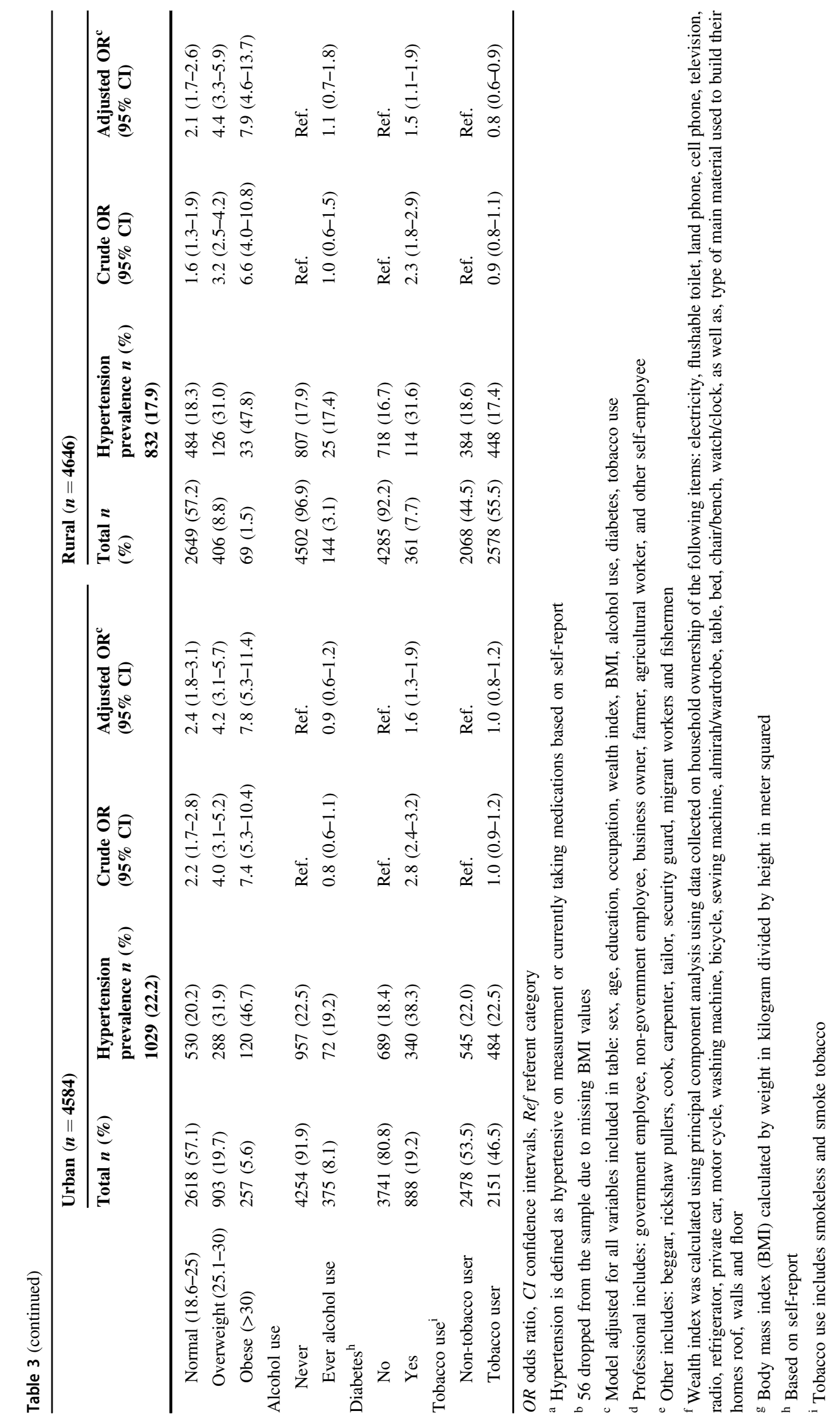


hypertension among those who self-reported as unemployed or retired was $80 \%$ (aOR: 1.8, 95\% CI: 1.3-2.6) higher when compared to those with professional employment. Additionally, for rural individuals with diabetes the odds of having hypertension were $50 \%$ (aOR: $1.5,95 \%$ CI: $1.1-1.9$ ) higher than those without diabetes.

\section{Prevalence and risk factors for pre-hypertension}

Table 4 presents the prevalence and risk factors for prehypertension by area of residence, excluding those with existing hypertension at study measurement $(n=7368)$. Among urban and rural residents, $44 \%$ and $41.5 \%$ were categorized as pre-hypertensive, respectively. Among both urban and rural participants, odds of pre-hypertension increased as age and BMI increased. The odds of prehypertension increased by $20 \%$ (aOR: 1.2, 95\% CI: 1.0-1.5) among those with diabetes from urban areas. Additionally, the odds of pre-hypertension among urban day laborers and industrial workers was $26 \%$ lower when compared to those professionally employed (aOR: 0.7, 95\% CI: $0.6-1.0$ ). Urban participants in the 4th wealth quartile had $50 \%$ higher odds of pre-hypertension compared to those in the 1st wealth quartile. Among rural participants, the odds of pre-hypertension increased by $70 \%$ among those who are unemployed or retired when compared to the professionally employed. Additionally, the odds of pre-hypertension increased as wealth index increased among rural participants.

\section{Discussion}

Utilizing data from this nationally representative sample, we found that one in five Bangladeshi adults (aged 25 years and above) have hypertension and that the prevalence of hypertension among this population increases both with increasing age and BMI. Diabetes significantly increases the odds of developing hypertension by almost $70 \%$ and $50 \%$ among urban and rural populations, respectively. Twelve percent of the population had previously been diagnosed with hypertension or high blood pressure by a health-care provider, however, only half of these adults self-reported to take medication to control their symptoms. Additionally, almost three-quarters of those who self-reported to have been previously diagnosed with hypertension continued to have uncontrolled hypertension at the time of study measurement. The majority of participants with uncontrolled hypertension were older in age and female.

Through this survey, we identified a higher prevalence of hypertension in urban than in rural populations (20\% vs. $16 \%$ ). This finding is similar to previously published findings, however, prevalence of hypertension appears to be lower in our study sample: The 2011 Bangladesh
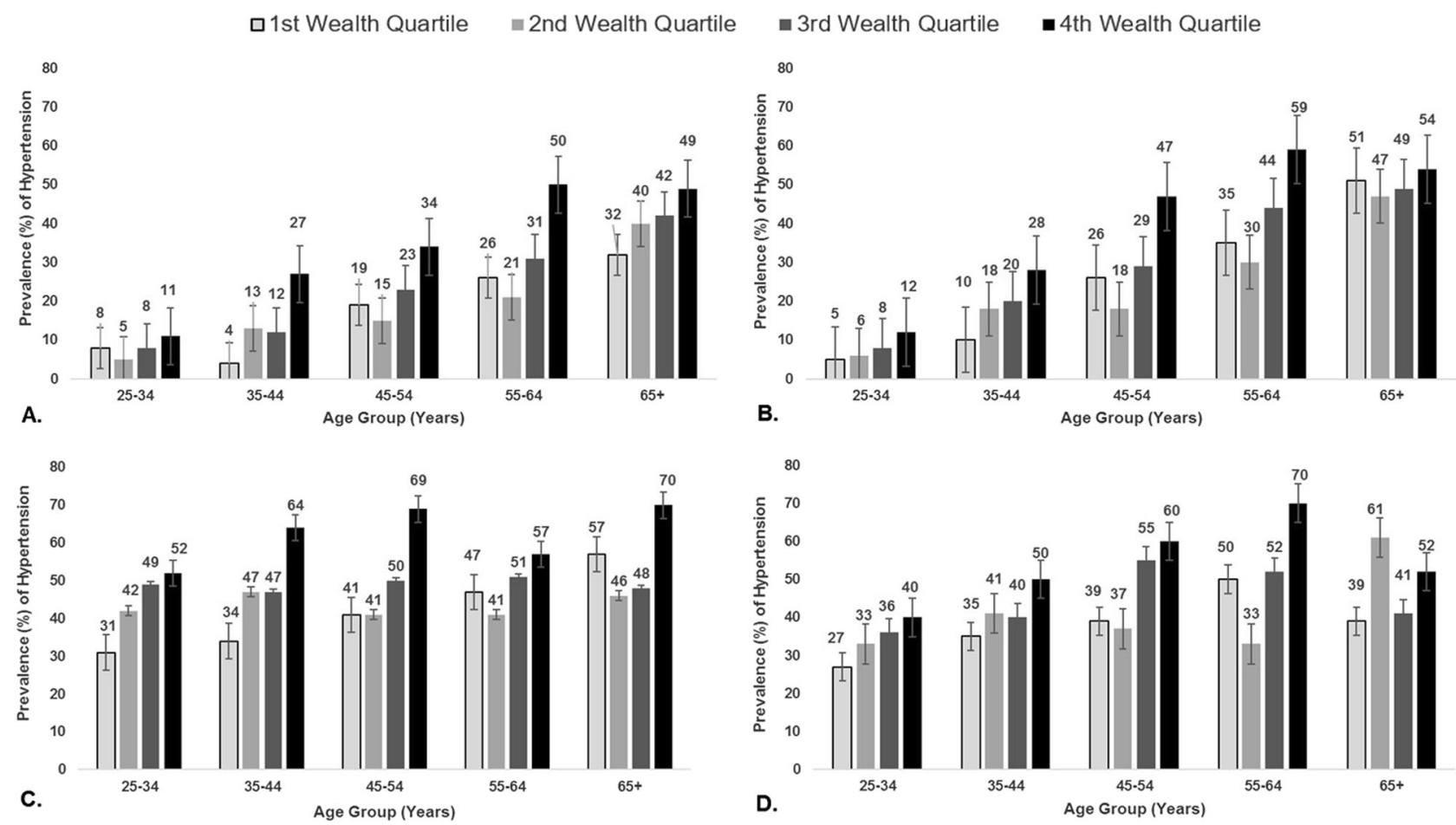

Fig. 2 Prevalence of Hypertension among males (a) Hypertension among females (b) Pre-hypertension among males (c), and Pre-hypertension among females (d) by Wealth Index and age group among 9275 Bangladeshi adults, 2010 
Demographic and Health Survey found that the overall prevalence of hypertension was $24.4 \%$, with urban residents as high as $32.7 \%$ and rural residents $23.2 \%$ [14]. However, this higher prevalence in hypertension may be attributable to the older study population of 35 years and above compared to the current study. Other studies with similarly aged study participants have found the prevalence of hypertension to range from 13 to $23.7 \%$ [12, 13, 20]. A metaanalysis of hypertension studies in Bangladesh conducted between 1994 and 2002 reported $13.5 \%$ as a pooled estimate of hypertension prevalence [21], which is lower than our estimated prevalence. This lower prevalence of hypertension, however, may be attributed to the time period these studies were sampled from and further indicates the rise in prevalence of hypertension based on this population-based study.

In our sample, $12 \%$ of participants reported to have previously been diagnosed with hypertension by a healthcare provider. Of particular concern, is that over half of participants who knew of their diagnosis continued to have hypertension at study measurement. In fact, those of age 65 years and above had the highest proportion of uncontrolled hypertension; $65 \%$ of males and $77 \%$ of females over the age of 65 years continued to have hypertension at study measurement despite their previous diagnosis. Reasons for poor adherence to hypertension medication have not been explored in this population. However, it has been studied extensively in the West. Previous studies have shown that the two most important factors contributing to poor adherence are the asymptomatic and chronic nature of the disease. Other factors associated with adherence include, demographic factors such as age, the patient's understanding and perception of hypertension, the health-care provider's model of delivering treatment, and the relationship between patients and health-care professionals [22, 23].
These factors indicate that a patient's experience with their primary health-care provider is key to ensuring successful treatment. However, Bangladesh has a stark shortage in health-care providers, particularly primary care [24]. Community health workers (CHW) have been utilized in the past to address this shortage and have been successful in improving coverage in vaccination and oral rehydration therapy programs [25]. Community programs that have successfully targeted infectious diseases should be adapted to address chronic diseases and provide the ongoing care needed to manage these lifelong diseases. The rising burden of NCDs along with the shortage of primary health-care providers is a serious concern for Bangladesh's health systems, particularly now as the country is moving toward universal health coverage. Access and availability to essential NCD prevention and treatment services remain fragmented [26]. Novel approaches will be necessary to ensure patients are kept within the health-care system and these approaches will have to be adapted to both urban and rural settings. Previous studies have shown that health workers without formal medical education can be adequately trained to effectively screen for and identify people at risk for cardiovascular diseases in Bangladesh [27]. Efforts should be made to develop and deploy CHWs into the field to effectively identify, screen, and treat adults with pre-hypertension to prevent chronic hypertension and ultimately, CVDs.

To identify those at highest risk of developing hypertension, we evaluated the prevalence of pre-hypertension and associated risk factors. This analysis provides the first assessment of risk factors of pre-hypertension in urban areas of Bangladesh and adds to a previous study [28] conducted in rural populations. We found the prevalence of prehypertension to be $44.5 \%$ among urban populations and $41.5 \%$ among rural populations, which is higher than
Fig. 3 Prevalence of hypertension at study measurement among those previously diagnosed $(n=1156)$ (a) and not previously diagnosed $(n=8119)$ (b) by sex and age group among 9275 Bangladeshi adults, 2010

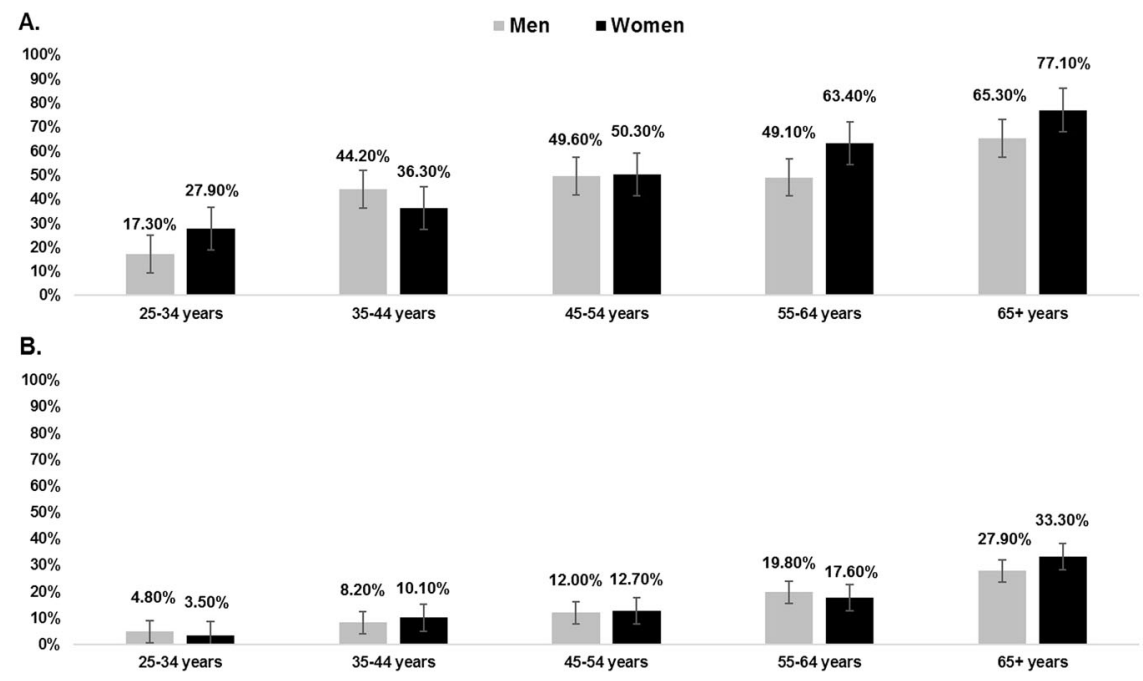




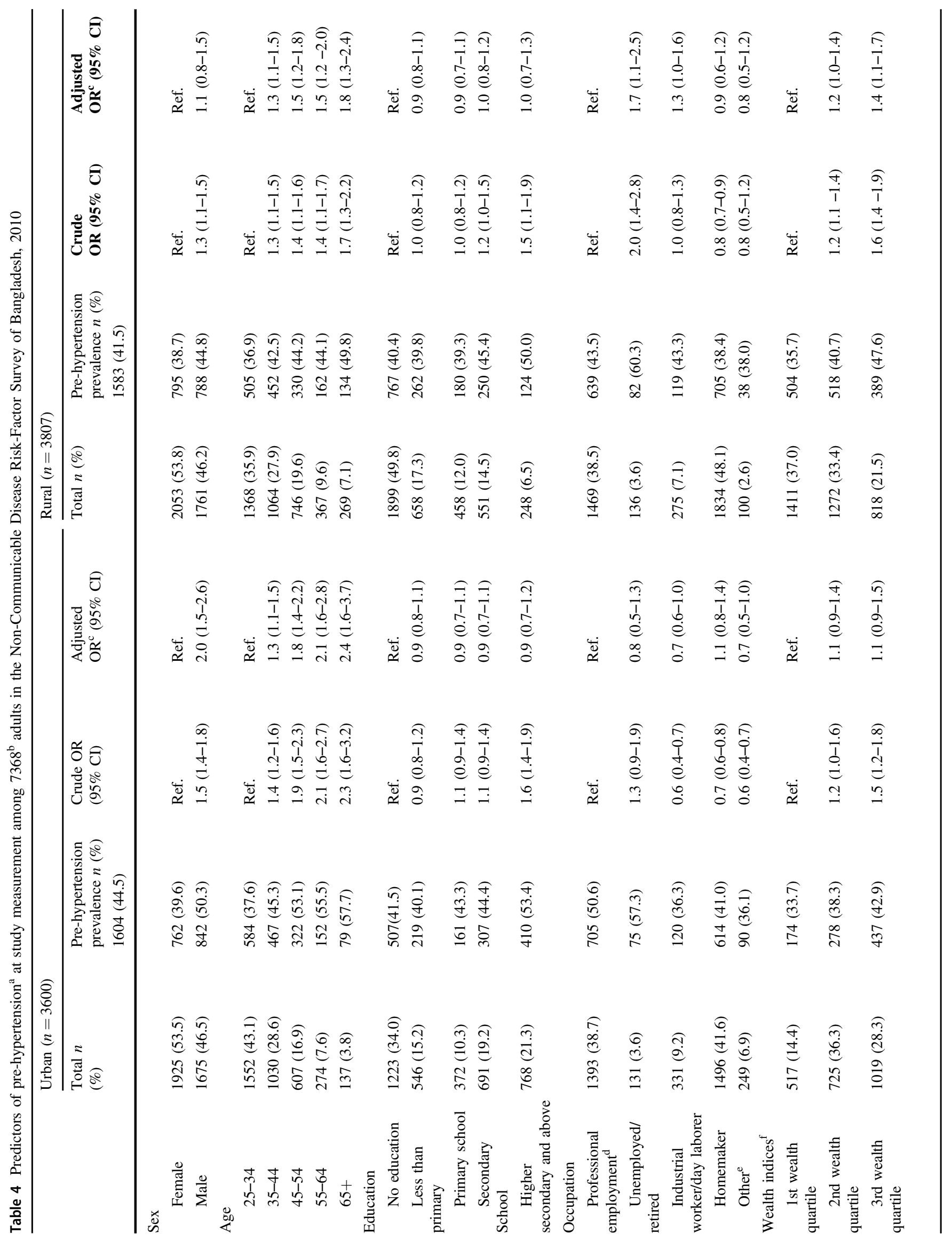




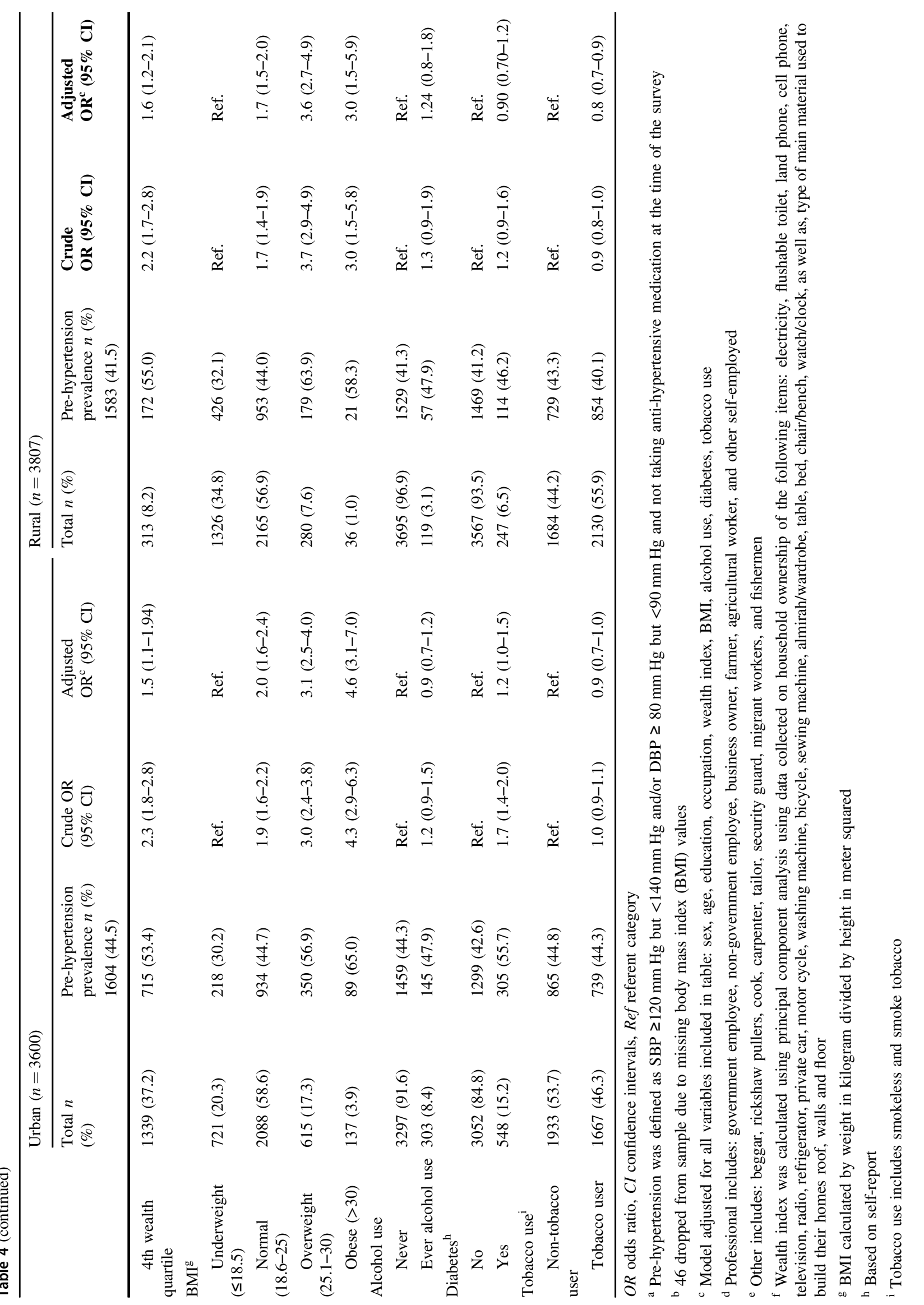


previously documented. In both urban and rural areas, males had a higher prevalence of pre-hypertension than females, which is consistent with previous findings [28-30]. Among both the urban and rural population, male gender, older age, higher BMI, and higher secondary and above educational status were found to be significant predictors of pre-hypertension. Diabetes was found to be a significant risk factor of pre-hypertension among urban residents only. Based on these findings, a preventive and predictive approach to the prevention of both hypertension and prehypertension can be employed, including community-based strategies and interventions directed at high-risk sub-groups identified through this multivariable analysis.

This study has several strengths. The main strength of this survey is the large sample size, coverage, representativeness of the national population, and the novel data it provides on management of hypertension among the general adult population of Bangladesh. Data collected through this survey were comprehensive and included diet, comorbidities, tobacco use and various demographic variables. Blood pressure measurements and current hypertension status were determined by trained and experienced health technicians using WHO-recommended methods rather selfreporting. Standard and valid data collection tools were utilized during blood pressure measurements, reducing the potential for measurement error and bias. However, several limitations should also be considered when interpreting the results of this analysis. The cross-sectional nature of this study design limits our ability to establish a causal relationship between the observed associations and hypertension or pre-hypertension. We were unable to measure factors such as family history of hypertension, and obtain blood samples to measure factors such as HDL-cholesterol levels and glycosylated immunoglobulin-G [31], which are important risk factors for hypertension and prehypertension.

Based on a recent meta-analysis, the association between pre-hypertension and an increased risk of coronary heart disease (CHD) has been established [32]. Pre-hypertension is recognized as an important public health problem and an opportunity for prevention of chronic outcomes. A preventive approach should be taken with individuals identified to be pre-hypertensive should be monitored more closely to monitor BP since a significant proportion of them are likely to develop hypertension [33]. Pre-hypertension as a causative risk factor is still debated as those found to be prehypertensive should not be subject to any specific treatment rather recommended to make lifestyle adjustments, such as reduce BMI, be more active, and improve nutritional habits [34]. Further research is needed to assess the benefit of short-term pharmacologic interventions to reduce the risk of CVD among pre-hypertensive patients, specifically among those of Asian descent [34].

\section{Conclusion}

In conclusion, our results show a high prevalence of hypertension and pre-hypertension in the population. Risk factors for both hypertension and pre-hypertension mainly included older age, high BMI, and having diabetes. Although age and gender are un-modifiable factors, care should be taken among these populations to focus public health interventions on modifiable behavioral factors such as weight, physical activity, and diet. A preventive and predictive community-based approach directed at those identified to be pre-hypertensive, those at highest risk of hypertension, should be employed to control the future burden of NCDs in Bangladesh. The primary health-care system of Bangladesh is limited due to a shortage of health-care personnel, including doctors, physicians, pharmacists, and other primary care providers [35]. To achieve the WHO's Global NCD Monitoring Framework's target of $25 \%$ reduction in preventable deaths from CVDs by 2025 [36], the health system of Bangladesh will need to be developed and transformed to provide personalized medical care with a focus on self-management to effectively manage risk factors and comorbidities.

\section{Summary}

\section{What is known about this topic?}

- Few national-based studies to assess prevalence of hypertension and pre-hypertension among Bangladesh adults have been conducted.

- High body mass index, age, area of residence, and gender are known risk factors for hypertension in Bangladesh.

- Hypertension is largely untreated in Bangladesh.

\section{What this study adds?}

- This study provides national population-based data on prevalence of hypertension and pre-hypertension among Bangladeshi adults.

- This study is the first to report national-based prevalence of pre-hypertension and identify risk factors among adults aged 25 and above in Bangladesh.

- This study provides further support that national efforts should be made to improve treatment among hypertensive patients, and also provides novel insights into potential target groups for preventive programs.

Author contributions JYI analyzed the data, wrote the manuscript; MMZ designed the study and sampling strategy, guided data analysis, interpreted data critically, and revised the manuscript; MMR, MAJC, HNA, MRR, MMH, MZH, MBA and RY designed the study, 
developed questionnaire, completed field execution, coordinated all seven divisions, reviewed the manuscript.

Funding The study was done with technical and financial assistance of WHO Country Office for Bangladesh.

\section{Compliance with ethical standards}

Conflict of interest The authors declare that they have no competing interests.

Ethical approval Ethics approval and consent to participate: Ethical clearance was obtained from Bangladesh Medical Research Council (BMRC). Before the interview was conducted, written (or thumb print) consent was obtained from each participant in Bangla as per BMRC guidelines.

Informed consent Consent for publication: All authors have reviewed this manuscript and consented to its publication.

Open Access This article is licensed under a Creative Commons Attribution-NonCommercial-ShareAlike 4.0 International License, which permits any non-commercial use, sharing, adaptation, distribution and reproduction in any medium or format, as long as you give appropriate credit to the original author(s) and the source, provide a link to the Creative Commons license, and indicate if changes were made. If you remix, transform, or build upon this article or a part thereof, you must distribute your contributions under the same license as the original. The images or other third party material in this article are included in the article's Creative Commons license, unless indicated otherwise in a credit line to the material. If material is not included in the article's Creative Commons license and your intended use is not permitted by statutory regulation or exceeds the permitted use, you will need to obtain permission directly from the copyright holder. To view a copy of this license, visit http://creativecommons. org/licenses/by-nc-sa/4.0/.

\section{References}

1. A global brief on Hypertension. Silent Killer, global public health crisis. Geneva, Switzerland: World Health Organization; 2013.

2. Kearney PM, Whelton M, Reynolds K, Muntner P, Whelton PK, He J. Global burden of hypertension: analysis of worldwide data. Lancet (London, England). 2005;365:217-23. doi: 10.1016/ S0140-6736(05)17741-1. PubMed PMID: 15652604

3. Lim SS, Vos T, Flaxman AD, Danaei G, Shibuya K, AdairRohani $\mathrm{H}$, et al. A comparative risk assessment of burden of disease and injury attributable to 67 risk factors and risk factor clusters in 21 regions, 1990-2010: a systematic analysis for the Global Burden of Disease Study 2010. Lancet (London, England). 2012;380:2224-60. doi: 10.1016/S0140-6736(12)61766-8. PubMed PMID: 23245609; PubMed Central PMCID: PMCPMC4156511.

4. Forouzanfar MH, Liu P, Roth GA, Ng M, Biryukov S, Marczak L, et al. Global burden of hypertension and systolic blood pressure of at least 110 to $115 \mathrm{mmHg}, 1990-2015$. JAMA. 2017;317:165-82. https://doi.org/10.1001/jama.2016.19043. PubMed PMID: 28097354

5. Qureshi AI, Suri MF, Kirmani JF, Divani AA, Mohammad Y. Is prehypertension a risk factor for cardiovascular diseases? Stroke 2005;36:1859-63. https://doi.org/10.1161/01.STR.0000177495. 45580.f1. PubMed PMID: 16081866
6. Lloyd-Sherlock P, Beard J, Minicuci N, Ebrahim S, Chatterji S. Hypertension among older adults in low- and middle-income countries: prevalence, awareness and control. Int J Epidemiol. 2014;43:116-28. https://doi.org/10.1093/ije/dyt215. PubMed PMID: 24505082; PubMed Central PMCID: PMCPMC3937973

7. Abegunde DO, Mathers CD, Adam T, Ortegon M, Strong K. The burden and costs of chronic diseases in low-income and middle-income countries. Lancet (London, England). 2007;370:1929-38. doi: 10.1016/S0140-6736(07)61696-1. PubMed PMID: 18063029

8. Joshi P, Islam S, Pais P, Reddy S, Dorairaj P, Kazmi K, et al. Risk factors for early myocardial infarction in South Asians compared with individuals in other countries. JAMA. 2007;297:286-94. https://doi.org/10.1001/jama.297.3.286. PubMed PMID: 17227980

9. Malik A. Congenital and acquired heart diseases: (A survey of 7062 persons). Bangladesh Med Res Counc Bull. 1976;2:115-9. PubMed PMID: 1037368

10. Chowdhury MA, Uddin MJ, Haque MR, Ibrahimou B. Hypertension among adults in Bangladesh: evidence from a national cross-sectional survey. BMC Cardiovasc Disord. 2016;16:22 https://doi.org/10.1186/s12872-016-0197-3. PubMed PMID: 26809175; PubMed Central PMCID: PMCPMC4727356

11. Islam FM, Bhuiyan A, Chakrabarti R, Rahman MA, Kanagasingam Y, Hiller JE. Undiagnosed hypertension in a rural district in Bangladesh: The Bangladesh Population-based Diabetes and Eye Study (BPDES). J Hum Hypertens. 2016;30:252-9. https:// doi.org/10.1038/jhh.2015.65. Epub 2015/06/26PubMed PMID: 26108363

12. Islam SM, Mainuddin A, Islam MS, Karim MA, Mou SZ, Arefin $S$, et al. Prevalence of risk factors for hypertension: A crosssectional study in an urban area of Bangladesh. Global Cardiol. Sci. Pract. 2015;2015:43 https://doi.org/10.5339/gcsp.2015.43. Epub 2016/01/19PubMed PMID: 26779518; PubMed Central PMCID: PMCPmc4710872.

13. Khanam MA, Lindeboom W, Koehlmoos TL, Alam DS, Niessen L, Milton AH. Hypertension: adherence to treatment in rural Bangladesh--findings from a population-based study. Global Health Action. 2014;7:25028 https://doi.org/10.3402/gha.v7. 25028. Epub 2014/11/02PubMed PMID: 25361723; PubMed Central PMCID: PMCPmc4212079

14. Rahman MM, Gilmour S, Akter S, Abe SK, Saito E, Shibuya K. Prevalence and control of hypertension in Bangladesh: a multilevel analysis of a nationwide population-based survey. J Hypertens. 2015;33:465-72. https://doi.org/10.1097/hjh. 0000000000000421. discussion 72. Epub 2014/11/08PubMed PMID: 25380166

15. Umemura S. [Significance of treating prehypertension with an angiotensin-receptor blocker--results from TROPHY study]. Nihon Rinsho. 2008;66:601-9. PubMed PMID: 18326331

16. Chronic Disease and Health Promotion. STEP wise approach to surveillance (STEPS). Geneva: World Health Organization; 2017.

17. Global Adult Tobacco Survey (GATS), Bangladesh Report 2009. Dhaka: World Health Organization; 2009.

18. Zaman MM, Rahman MM, Rahman MR, Bhuiyan MR, Karim MN, Chowdhury MA. Prevalence of risk factors for noncommunicable diseases in Bangladesh: Results from STEPS survey 2010. Indian J Public Health. 2016;60:17-25. https://doi.org/ 10.4103/0019-557X.177290. PubMed PMID: 26911213

19. Surveillance of risk factors for noncommunicable disease. the WHO STEPwise approach. Geneva: World Health Organization; 2001.

20. Alam DS, Chowdhury MA, Siddiquee AT, Ahmed S, Niessen LW. Awareness and control of hypertension in Bangladesh: follow-up of a hypertensive cohort. BMJ Open. 2014;4:e004983 
https://doi.org/10.1136/bmjopen-2014-004983. PubMed PMID: 25537780; PubMed Central PMCID: PMCPMC4275678

21. Moniruzzaman TA, Rahman S. Prevalence of hypertension among the Bangladeshi adult population: a meta-analysis. Reg Health Forum. 2013;17:15-9.

22. Ruilope LM. Long-term adherence to therapy: the clue to prevent hypertension consequences. Eur Heart J. 2013;34:2931-2. https:// doi.org/10.1093/eurheartj/eht299. PubMed PMID: 23990601

23. Botelho RJ, Skinner H. Motivating change in health behavior. Implications for health promotion and disease prevention. Prim Care. 1995;22:565-89. PubMed PMID: 8668730

24. Afsana K, Grant JP, Evans J. Crisis of health workforce in Bangladesh: a non-government organization's experience in deploying community health workers in primary health care. Perspect Public Health. 2014;134:253-4. https://doi.org/10.1177/ 1757913914545291. PubMed PMID: 25169609

25. Chowdhury AM, Bhuiya A, Chowdhury ME, Rasheed S, Hussain Z, Chen LC. The Bangladesh paradox: exceptional health achievement despite economic poverty. Lancet (London, England). 2013;382:1734-45. doi: 10.1016/S0140-6736(13)62148-0. PubMed PMID: 24268002

26. Ahmed S, Chowdhury MA, Khan MA, Huq NL, Naheed A. Access to primary health care for acute vascular events in rural low income settings: a mixed methods study. BMC Health Serv Res. 2017;17:47 https://doi.org/10.1186/s12913-017-1987-8. PubMed PMID: 28100208; PubMed Central PMCID: PMCPMC5242000

27. Gaziano TA, Abrahams-Gessel S, Denman CA, Montano CM, Khanam M, Puoane T, et al. An assessment of community health workers' ability to screen for cardiovascular disease risk with a simple, non-invasive risk assessment instrument in Bangladesh, Guatemala, Mexico, and South Africa: an observational study. Lancet Glob Health. 2015;3:e556-63. doi: 10.1016/S2214-109X (15)00143-6. PubMed PMID: 26187361; PubMed Central PMCID: PMCPMC4795807

28. Khanam MA, Lindeboom W, Razzaque A, Niessen L, Milton AH. Prevalence and determinants of pre-hypertension and hypertension among the adults in rural Bangladesh: findings from a community-based study. BMC Public Health. 2015;15:203 https:// doi.org/10.1186/s12889-015-1520-0. PubMed PMID: 25880433; PubMed Central PMCID: PMCPMC4355126
29. Wu Y, Huxley R, Li L, Anna V, Xie G, Yao C, et al. Prevalence, awareness, treatment, and control of hypertension in China: data from the China National Nutrition and Health Survey 2002. Circulation. 2008;118:2679-86. https://doi. org/10.1161/CIRCULATIONAHA.108.788166. PubMed PMID: 19106390

30. Tsai PS, Ke TL, Huang CJ, Tsai JC, Chen PL, Wang SY, et al. Prevalence and determinants of prehypertension status in the Taiwanese general population. J Hypertens. 2005;23:1355-60. PubMed PMID: 15942457

31. Wang Y, Klaric L, Yu X, Thaqi K, Dong J, Novokmet M, et al. The association between glycosylation of immunoglobulin $\mathrm{G}$ and hypertension: A multiple ethnic cross-sectional study. Medicine. 2016;95:e3379 https://doi.org/10.1097/MD.0000000000003379. PubMed PMID: 27124023; PubMed Central PMCID: PMCPMC4998686

32. Huang Y, Cai X, Liu C, Zhu D, Hua J, Hu Y, et al. Prehypertension and the risk of coronary heart disease in Asian and Western populations: a meta-analysis. J Am Heart Assoc. 2015;4: https://doi.org/10.1161/JAHA.114.001519. PubMed PMID: 25699996; PubMed Central PMCID: PMCPMC4345875.

33. Chobanian AV, Bakris GL, Black HR, Cushman WC, Green LA, Izzo JL,Jr, et al. The Seventh Report of the Joint National Committee on Prevention, Detection, Evaluation, and Treatment of High Blood Pressure: the JNC 7 report. JAMA. 2003;289:2560-72. doi:https://doi.org/10.1001/jama.289.19. 2560PubMed PMID: 12748199.

34. Habib GB, Virani SS, Jneid H. Is 2015 the primetime year for prehypertension? Prehypertension: a cardiovascular risk factor or simply a risk marker? J Am Heart Assoc. 2015;4: https://doi.org/ 10.1161/JAHA.115.001792. PubMed PMID: 25699997; PubMed Central PMCID: PMCPMC4345880.

35. Zaman MMUA, Bhuiyan MR, Karim MN, Moniruzzaman RahmanSMA. Noncommunicable Disease Prevention and Control Situation in a Primary Health Care Setting of Bangladesh: Design and Baseline Findings of an Intervention. Chronic Dis. Int. 2016;3:1-6.

36. NCD Global Monitoring Framework: World Health Organization; 2017 [cited 2017 09/29]. Available from: http://www.who.int/ nmh/global_monitoring_framework/en/. 